\title{
Chest pain in emergency department: A diagnosis of diabetic ketoacidosis must be ruled out
}

\author{
Nasir Mohamad, Rashidi Ahmad, PK Cheah
}

\begin{abstract}
Introduction: Diabetic ketoacidosis (DKA) is a common diabetic complication presenting to the Emergency Department (ED). Early recognition and initial aggressive treatment of DKA decreases morbidity and mortality. Clinical presentations of DKA are non specific such as nausea, vomiting, dehydration and abdominal pain. Chest pain is unusual presentation of DKA, however, acute coronary syndrome and pericarditis that manifest with chest pain are known precipitating factors of DKA. Case Report: We report a case of a middle aged diabetic patient who was presented with severe chest pain and elevated creatine kinase that might have thrown us off the correct diagnosis of DKA. Conclusion: A description of his presentations and acute management, along with review of literatures, is presented.
\end{abstract}

Nasir Mohamad ${ }^{1}$, Rashidi Ahmad ${ }^{2}$, PK Cheah ${ }^{3}$

Affiliations: ${ }^{1}$ Senior Lecturer/ Consultant Emergency Physician, Department of Emergency Medicine, School of Medical Sciences, 16150 Kubang Kerian, Kelantan, Malaysia; ${ }^{2}$ Senior Lecture/ Emergency Physician, Department of Emergency Medicine, School of Medical Sciences, Health Campus USM, 16150 Kubang Kerian, Kelantan, Malaysia; ${ }^{3}$ Emergency Physician, Department of Emergency Medicine, School of Medical Sciences, Health Campus USM, 16150 Kubang Kerian, Kelantan, Malaysia.

Corresponding Author: Nasir Mohamad, Department of Emergency Medicine, School of Medical Sciences, 16150 Kubang Kerian, Kelantan, Malaysia; Phone: +6097676978; Fax: +6097673219;

Email: drnasirmohamadkb@yahoo.com

Received: 05 September 2010

Accepted: 19 October 2010

Published: 15 November 2010
Keywords: Chest pain, Elevated creatine kinase, Diabetic ketoacidosis

$* * * * * * * * *$

Mohamad N, Ahmad R, Cheah PK. Chest pain in emergency department: A diagnosis of diabetic ketoacidosis must be ruled out. International Journal of Case Reports and Images 2010;1(3):6-9.

$$
* * * * * * * * * \text {. }
$$

doi:10.5348/ijcri-2010-11-5-CR-2

\section{INTRODUCTION}

Acute coronary syndrome (ACS) and DKA are common medical emergencies. They have different clinical manifestations. The main symptom of ACS is chest pain. However, patients may present with atypical chest pain or angina equivalent such as exertion dyspnoea. In contrast, DKA has varied clinical symptoms. The typical symptoms include nausea and vomiting, abdominal pain, weight loss, dehydration, hypotension, tachycardia, Kausmaul's respiration and odour of acetone on the breath [1]. The non-typical symptoms have been reported such as DKA associated with pericarditis and myocardial . However, the mechanism in the development of myocardial necrosis remains unclear [2-4].

Many patients presenting with severe chest pain in Emergency Department (ED) initially believed to be cardiac in aetiology may, in fact, have diabetic ketoacidosis (DKA) as an alternative or additional cause of their complaints. We describe a known diabetic patient who is presented to ED with severe chest pain and elevated creatine kinase might have thrown us off the correct diagnosis of DKA. 


\section{CASE REPORT}

A 44-year-old man arrived at the Emergency Department (ED) triage counter screaming in pain and holding his chest. He was immediately sent to the red zone. We were unable to get a proper history as he repeatedly complained of severe central chest pain and shortness of breath. Initial focused examination revealed he was in severe chest pain, restless, moderately dehydrated, dyspneic and tachypnoeic. His BMI was 26.2. His initial blood pressure, heart rate, respiratory rate, oxygen saturation and temperature were $129 / 62 \mathrm{mmHg}, 98$ beats per minute, 28 per minute, $100 \%$ under room air and $37^{\circ} \mathrm{C}$ respectively. Electrocardiogram (ECG) revealed sinus tachycardia rhythm. All peripheral pulses were palpable and equal on both sides. Abdominal examination revealed soft abdomen with mild tenderness over the epigastric area. Bowel sounds were normal (10/min). Examinations of the cardiovascular and respiratory system were unremarkable.

High flow oxygen via non rebreathing mask was instituted and an intravenous drip of normal saline according to the protocol was initiated. Soluble aspirin $300 \mathrm{mg}$, sublingual glyceryl trinitrate (GTN) $0.5 \mathrm{mg}$ and isosorbide dinitrate infusion $10 \mathrm{mcg} / \mathrm{min}$ were administered. The chest pain was persistent despite the initial treatment. The pain was controlled after 10 mg of intravenous morphine (titrating dose every 5 minutes). Intravenous midazolam of $2.5 \mathrm{mg}$ was also administered to make him more calm and comfortable. He was treated as acute coronary syndrome (ACS). Intravenous heparin according to the protocol was administered.

Further history was obtained after the pain was controlled; it was sudden in onset, about one hour prior to admission. The patient claimed that he had intermittent, sudden central chest pain, radiating to the neck and epigastric area, lasting for a few minutes, and relieved spontaneously for the past two days. He had difficulty in describing the nature of pain. The chest pain was associated with difficulty in breathing and sweating. The pain was not precipitated by breathing, exercise and food intake. He had a few occasions of vomiting and low-grade fever for the last 3 days. He did not seek any treatment from a general practitioner. He has been suffering from Type II diabetes mellitus since 5 years and is currently on subcutaneous Actrapid insulin (18 IU/18U/10U). For the past 2 weeks of fasting month-Ramadhan, he did not take his daylight insulin injections since he was fasting and he was afraid that insulin might cause him hypoglycemia. He is a non-smoker and has no history of ischemic heart disease or hypertension. However, he had family history of heart ailments and hypertension in his first degree relative.

Serial electrocardiograms showed a normal sinus tachycardia and absence of ST-T changes. Bedside laboratory tests demonstrated hyperglycemia (capillary blood glucose $17.1 \mathrm{mmol} / \mathrm{L}$ ) and wide anion gap metabolic acidosis (arterial blood gases $\mathrm{pH}, 7.24$; bicarbonate, $13.1 \mathrm{mmol} / \mathrm{L}$; paCO2, $25.1 \mathrm{mmHg}$; pO2, $315 \mathrm{mmHg}$; BE, -10 and sodium, $133 \mathrm{mmol} / \mathrm{L}$; potassium, $4.7 \mathrm{mmol} / \mathrm{L}$; urea $4.7 \mathrm{mmol} / \mathrm{L}$; chloride, 93 $\mathrm{mmol} / \mathrm{L}$; anion gap, $27 \mathrm{mmol} / \mathrm{L})$. Urine analysis demonstrated heavy ketonuria dipstick urine ketone, $4+$, glycosuria (urine sugar +++ ) and absence of urinary tract infection (urine nitrite and leukocyte nil) and rhabdomyolysis (haemoglobinuria or tea coloured urine). His full blood counts showed haemoglobin, $13.6 \mathrm{~g} / \mathrm{dL}$; total white cell count, $17,400 / \mathrm{uL} \quad(72.3 \%$ neutrophils and $22.2 \%$ lymphocytes); platelet count, 280,000/uL. His creatine kinase (CK) during admission was $879 \mathrm{U} / \mathrm{L}$. Troponin $\mathrm{T}$ during admission was normal $(\mathrm{T}<0.01$ $\mu \mathrm{g} / \mathrm{L})$. Chest radiograph showed normal lung field and no cardiomegaly. Supine plain abdominal radiograph showed normal distribution of gases and no calcification seen.

A differential diagnosis of Diabetic Ketoacidosis (DKA) precipitated by acute coronary syndrome was made based on the clinical findings (chest pain) and laboratory results (hyperglycemia, heavy ketonuria, wide anion gap metabolic acidosis and elevated CK). Rehydration therapy and subcutaneous injections of soluble insulin (Actrapid 6 IU/hour) were started. His condition was getting better after $2.5 \mathrm{~L}$ of normal saline infusion. His blood glucose, blood pressure and heart rate after two hours in ED were $13.2 \mathrm{mmol} / \mathrm{L}$, $110 / 80 \mathrm{mmHg}$ and 80 beats per minute respectively. His arterial blood gases showed some improvement (pH: 7.31; bicarbonate, $20.3 \mathrm{mmol} / \mathrm{L}$; paCO2, 38.5 $\mathrm{mmHg}$; pO2, $125 \mathrm{mmHg}$; $\mathrm{BE},-5.7$. The patient was transferred to the medical ward for further management and observation.

Serial electrocardiogram (ECG) showed no evidence of ischemic heart disease (ST-T changes). Serial creatine kinase (CK) (normal value: < $190 \mathrm{U} / \mathrm{L}$ ) on day 1, day 2 and day 3 were $879 \mathrm{U} / \mathrm{L}, 751 \mathrm{U} / \mathrm{L}$ and $579 \mathrm{U} / \mathrm{L}$ respectively. Serial CK-MB (normal value: < $25 \mathrm{U} / \mathrm{L}$ ) on day 1, day 2 and day 3 were $46 \mathrm{U} / \mathrm{L}, 39 \mathrm{U} / \mathrm{L}$ and 28 $\mathrm{U} / \mathrm{L}$ respectively. Qualitative Troponin $\mathrm{T}$ six hours apart was less than $0.01 \mu \mathrm{g} / \mathrm{L}$.

The chest pain improved after intensive fluid therapy and insulin infusion. His glucose level and metabolic acidosis returned to normal. Insulin infusion was tapered off 4 days later. He started ambulating without any further complaints of chest pain. According to the Cardiologist, there was no objective evidence to suggest his current illness was due to acute coronary syndrome. Hence, stress test and coronary angiography were not required at that point in time. Counselling on compliance to medication was given prior to discharge on day 10 of admission. He was given an outpatient appointment in the physician clinic for follow-up treatment. During recent follow-up, he denied chest pain and shortness of breath. His blood glucose was fairly controlled (FBS: $8.6 \mathrm{mmol} / \mathrm{L}$, HbA1c: 6.8\%). 


\section{DISCUSSION}

Management of patients with severe chest pain is a great challenge to the emergency residents. Adequate history related to the symptom may be difficult to obtain from such patients on presentation. We strongly suggest an administration of titrated dose of intravenous narcotic to relief pain suffering once primary survey is completed and vital signs have been recorded and evaluated. Adequate pain relief facilitates history taking and improves patient satisfaction to hospital management.

The patient presented to us with a severe chest pain made the ACS the most likely diagnosis despite a normal ECG. Elevated creatine kinase made our suspicion of ACS greater and he was managed accordingly. It is known that diabetes is an important risk factor for coronary artery disease and it is associated with a worsened prognosis for patients with acute myocardial infarction. Therefore, diabetic patients who have chest pain either typical or atypical angina is considered to have high or moderate probability of ACS. Meanwhile, it has been reported that DKA and ACS may be presented together [5]. Routine practice of blood sugar checking and arterial blood gases by the medical personnel's for tachypneic diabetic patients did help in making a diagnosis of DKA at the resuscitation zone. The presence of hyperglycemia and metabolic acidosis warranted us to check for urine ketones that turned out to be significant.

Most of the clinical manifestations of DKA are related to metabolic derangements such as hyperglycemia and ketonemia. Nausea, vomiting and abdominal pains have been studied repeatedly and have been found to be present in up to $70 \%$ of patients with DKA [6]. The cause of these disturbances is unclear. Gastric dilatation, paralytic ileus, pancreatitis and stretching of the liver capsule or liver infarct may be present [1]. Chest pain is unusual presentation of DKA. Lactic acidosis induced cardiac chest pain might explain the cause of chest pain. Acidic pH evokes large inward currents in almost all cardiac sympathetic afferents and the biophysical properties of the acidevoked currents in cardiac afferents match the acidsensing ion channel 3 (ASIC3). The activated cardiac afferent neurons mediate the sensation of angina. The above findings were also noted in patients with myocardial ischemia.

Our patient had elevated CK and CK-MB level. However, Troponin T level was normal. False positive elevations for CK-MB may occur in patients with DKA, nonketotic hyperglycemia, muscular dystrophy, renal failure, rhabdomyolysis, prostate surgery and after strenuous athletic activity. Elevated creatine kinase in acute myocardial infarction is due to myocardial cell death/necrosis, whereas, in DKA it is due to rhabdomyolysis. Rhabdomyolysis occurs in as many as $50 \%$ of patients with DKA and varies in severity from mildly elevated CK levels with no symptoms to markedly elevated CK with acute renal failure, possibly requiring hemodialysis [7]. Rhabdomyolysis occurs commonly in patients with DKA but is usually subclinical. Rhabdomyolysis associated with DKA may be overlooked, resulting in renal failure that may be averted with appropriate therapy. The mechanism of DKA-mediated muscle injury is uncertain. Theories include insufficientenergy delivery to muscles, hyperosmolar effects and underlying metabolic defects such as McArdle's [7].

According to the WHO expert committee, a diagnosis of non-ST elevation acute coronary syndromes is made when patients have symptoms of ischemic chest pain and elevated cardiac enzymes [8]. Current consensus guidelines of the Joint European Society of Cardiology and American College of Cardiology state that troponins are the preferred biomarkers of myocardial necrosis; this is because of their improved sensitivity and specificity compared with the conventional biomarkers creatine kinase and its isoenzyme MB (CK-MB) [9]. In fact, in the setting of cardiac ischaemia or coronary, myocardial infarction (MI) is defined as a typical rise and fall of troponin; the alternative CK-MB is used only when Tn assays are not available.

DKA does cause acute myocardial necrosis [3]. However, the mechanism is unclear. Theories include severe acid-base and electrolyte disturbances triggered coronary spasms and dehydration, low cardiac output, increased blood viscosity, impaired blood flow, increased platelet aggregation, increased red blood cell rigidity and hemostatic changes triggering the development of thrombosis [10]. DKA results in a prothrombotic state and activation of the vascular endothelium, which in turn predispose to cerebrovascular accidents or myocardial necrosis [3].

\section{CONCLUSION}

Many patients presenting with severe chest pain in Emergency Department (ED) initially believed to be cardiac in aetiology may, in fact, have diabetic ketoacidosis (DKA) as an alternative or additional cause of their complaints. The prompt recognition of DKA and simple institution of rapid rehydration have continued to reduce the mortality and complications. It is advisable to perform random blood glucose and urine ketone in diabetic patients as the investigations are affordable and non invasive. As ACS is very difficult to rule out, in fact, it may be associated with carditis or myocarditis, we should consider cardiac enzymes including troponin $\mathrm{T}$ and creatine kinase, serial ECG, and echocardiography during acute phase. Perhaps in the future, Echocardiograhy Stress Test, Technetium Scan or Coronary Angiography should be done in high-risk patients to rule out coronary artery disease.

$* * * * * * * * *$ 


\section{Acknowledgement}

I would like to thank Dean, School of Medical Sciences, USM, Malaysia, for his support.

\section{Author Contributions}

Nasir Mohamad - Conception and design, Acquisition of data, Analysis and interpretation of data, Drafting the article, Critical revision of the article, Final approval of the version to be published

Rashidi Ahmad - Conception and design, Acquisition of data, Analysis and interpretation of data, Critical revision of the article, Final approval of the version to be published

PK Cheah - Acquisition of data, Drafting the article, Final approval of the version to be published

\section{Guarantor}

The corresponding author is the guarantor of submission.

\section{Conflict of Interest}

Authors declare no conflict of interest.

\section{Copyright}

(C) Nasir Mohamad et. al. 2010; This article is distributed under the terms of Creative Commons attribution 3.0 License which permits unrestricted use, distribution and reproduction in any means provided the original authors and original publisher are properly credited. (Please see www.ijcasereportsandimages.com /copyright-policy.php for more information.)

\section{REFERENCES}

1. Tintinalli JE, Ruiz E, Krome RL. Emergency Medicine: A comprehensive study guides. Fourth ed. 1996: Mc-Graw Hill.

2. Kannel WB, McGee DL. Diabetes and cardiovascular disease. The Framingham study. JAMA 1979;241:2035-2038.

3. Tretjak M, Verovnik F, Vujkovac B, SlemenikPusnik C, Noc M. Severe Diabetic Ketoacidosis Associated With Acute Myocardial Necrosis. Diabetes Care 2003;26:2959-2960.

4. Oki K, Matsuura W, Saito Y, Ono Y, Yanagihara K, Sueshiro M, et.al. Infective Endocarditis and Acute Purulent Pericarditis in a Patient with Hyperglycemia. Internal Medicine 2005;44:666670.

5. Özkaya EÇ, Özbek M, Bozkurt NÇ, Çakal E, Karbek B, Delibasi T. A case of diabetic ketoacidosis with ECG abnormalities mimicking acute coronary syndrome [Abstract]. European Congress of Endocrinology, 2010;22:270.

6. Umpierrez GE. Khajavi M, Kitabchi AE. Review: Diabetic ketoacidosis and hyperglycemic hyperosmolar nonketotic syndrome. Am J Med Sci 1996;311:225-233.

7. Chanson P, de Rohan-Chabot P, Loirat P, Lubetzki J. Nontraumatic rhabdomyolysis during diabetic ketoacidosis. Diabetologia 1986;29:229-234-234.
8. WHO, Hypertension and coronary heart disease: classification and criteria for epidemiological studies. Technical Report Series no. 168. 1959, Geneva: WHO.

9. Alpert JS, Thygesen K, Antman E, Bassand JP. Myocardial infarction redefined-a consensus document of The Joint European Society of Cardiology/American College of Cardiology committee for the redefinition of myocardial infarction: The Joint European Society of Cardiology/ American College of Cardiology Committee. J Am Coll of Cardiol 2000;36:959-969.

10. Carl GF, Hoffman WH, Passmore GG, Truemper EJ, Lightsey AL, Cornwell PE. Diabetic ketoacidosis promotes a prothrombotic state. Endocrine research 2003;29:73-82. 